\title{
ANÁLISIS MULTIRESOLUCIÓN
}

Luis Miguel Nuñez Ramirez ${ }^{1}$, Rodolto Galvez Perez ${ }^{1}$ \& Humberto Emiliano Galvez Perez ${ }^{1}$

Resumen: Antes de haberse formalizado el concepto del Análisis Multiresolución $(A M R)$, la construcción de bases ortonormales de ondículas debió ser demasiado dificultoso. Por ello, el estudio del $A M R$ adquiere importancia y por consiguiente la construcción de Ondículas se hace viable.

En la construcción de una ondicula $\Psi$, debemos determinar una función escala $\Phi$, para luego construir su Análisis Multiresolución asociado; en seguida se pasa ha construir $\Psi$, con propiedades deseadas para las aplicaciones.

Palabras clave: Análisis Multiresolución, Función escala, Base Ortonormal de Ondículas, Base de Riesz.

\section{ANALYSIS MULTIRESOLUTION}

Abstract: Before the concept has been formalized Analysis Multiresolution AMR, base construction orthonormal wavelet should be too difficult. For Therefore, the study of $A M R$ becomes important and therefore construction of Wavelets is viable.

In the construction of a wavelet $\Psi$, we determine a scale function $\Phi$, then build your Analysis Associated multiresolution; soon passed has built $\Psi$ with desired properties for applications.

Key words: Multiresolution Analysis, Function scale Orthonormal wavelet basis, Riesz base. 


\section{Introducción}

El argumento esencial de la teoría (en $\mathbb{R}$ ) es comenzar con una función, llamada Ondícula, generadora o Madre, la cual es sometida a encogimientos y alargamientos, asi como traslaciones por toda la recta, dando lugar al surgimiento de una familia de Ondícula, las que constituirán una base ortonormal del espacio en que se trabaja (en este caso $L^{2}(\mathbb{R})$ ).

Tal Ondícula, es concentrada en un cierto intervalo pero es movediza por toda la recta. Un ejemplo básico, pero importante son las Ondícula de A. Haar, aunque no son regulares. Sin embargo, es importante resaltar que antes de formalizarse el $A M R$, ya se habían construido ejemplos de bases ortonormales de Ondículas regulares.

\section{Análisis Multiresolución}

El concepto de Análisis Multiresolución $(A M R)$ de un espacio de Hilbert de funciones fue formulada por S. Mallat y Y. Meyer en 1986, lo que constituye un ejemplo de la armonía entre la matemática pura y la aplicada pués Mallat trabajaba en el análisis de la imagen, en donde es frecuente trabajar en forma simultánea en diferentes escalas, la cual motivo interpretar la bases ortonormales de Ondícula (construidas por Meyer) como instrumentos para escribir sistemá ticamente el incremento o disminución de información, lo cual permite obtener una buena ó mejor aproximación a altas escalas.

Definición 2.1. Un $A M R$ para $L^{2}(\mathbb{R})$ es una familia de subespacios cerrados $\left\{V_{n}\right\}_{n \in \mathbb{Z}}$ tales que:

i) $\ldots \subset V_{2} \subset V_{1} \subset V_{0} \subset V_{-1} \subset \ldots$

ii) $\bigcup_{n \in \mathbb{Z}} V_{n}$ es denso en $L^{2}(\mathbb{R})$.

iii) $\bigcap_{n \in \mathbb{Z}} V_{n}=\{0\}$.

iv) $V_{n+1}=D_{2} V_{n}=\left\{f\left(2^{-n} x\right): f(x) \in V_{n}\right\}$.

v) Existe $\phi \in V_{0}$ tal que $\left\{T_{n} \phi\right\}_{n \in \mathbb{Z}}$ es una base ortonormal (b.o.n) para $V_{0}$.

Observación 2.1. Apartir de iv) y v) se sigue que:

a) $f(x) \in V_{0} \Leftrightarrow f\left(2^{-m} x\right) \in V_{m}$.

b) $V_{0}$ es invariante por traslaciones, esto es,

$$
T_{k} V_{0}=\left\{f(x-k): f(x) \in V_{0}\right\}=V_{0}, k=0, \pm 1, \pm 2 \pm \cdots
$$

c) $V_{m}=D_{2^{m}} V_{0}=D_{2^{m}} T_{k} V_{0}=\left\{f\left(2^{-m} x-k\right): f(x) \in V_{0}\right\}$.

d) Para cada $m$ fijo, $\left\{\phi_{m, n}(x)=2^{-m / 2} \phi\left(2^{-m} x-n\right)\right\}_{m, n \in \mathbb{Z}}$ es una base ortonormal para $V_{m}$.

Ejemplo 2.1. Sea $V_{0}=\left\{f \in L^{2}(\mathbb{R}):\right.$ fes constante en $\left.[m, m+1), m \in \mathbb{Z}\right\}$

Nota:

$\phi(x)=\chi_{[0,1)},\left\{V_{m}=D_{2^{m}} V_{0}\right\}$ es un $A M R$. 
a) La definición de Mallat's de un $A M R$ es diferente pero equivalente al anterior. Su definición no estipula la existencia de la función $\phi$ y por lo tanto no incluye la condición $v$, pero establece dos condiciones más sobre los subespacios $\left\{V_{n}\right\}_{n \in \mathbb{Z}}$ :

i) Si $f(x) \in V_{n}$, entonces $f\left(x-2^{-n} k\right) \in V_{n}, \forall n \in \mathbb{Z}$.

ii) Existe un isomorfismo de $V_{0}$ sobre $\ell^{2}(\mathbb{Z})=\left\{c:\left\{c_{n}\right\}_{n \in \mathbb{Z}}, \sum_{n \in \mathbb{Z}}\left|c_{n}\right|^{2}<\infty\right\}$. Estas dos condiciones garantiza la existencia de $\phi$ y la validez de $v$ ).

b) La función $\phi$ es llamada la función escala de un $A M R$.

Ahora, surge la pregunta natural ¿Cómo un $A M R$ genera una base ortonormal de ondículas para $L^{2}(\mathbb{R})$ ?. Veamos, como $V_{n} \subset V_{n-1}$, definamos $W_{n}$ tal que

$$
V_{n-1}=V_{n} \bigoplus W_{n}, \forall n \in \mathbb{Z}
$$

Por inducción, se tiene que

$$
V_{n}=V_{n+k} \bigoplus_{i=1}^{k} W_{n+i}=V_{n+k} \bigoplus_{i=1}^{k} W_{n+i}
$$

donde todos los subespacios son ortogonales y

$$
\bigoplus_{i=1}^{n} W_{i}=W_{1} \bigoplus W_{2} \bigoplus \cdots \bigoplus W_{n}
$$

Debido a que $V_{n-1}=V_{n} \bigoplus W_{n}$, las condiciones $i i$ ) y iii) de la Definición 2.1, se tiene que

$$
L^{2}(\mathbb{R})=\bigoplus_{j=-\infty}^{\infty} W_{j}
$$

La familia de $\left\{W_{n}\right\}_{n \in \mathbb{Z}}$ presenta propiedades análogas como los $V_{n}$ 's. De este modo, debido a que $W_{n+1} \subset W_{n} ; V_{n+1}=\left\{f\left(2^{-1} x\right): f(x) \in V_{n}\right\}$ y $V_{n-1}=V_{n} \oplus W_{n}$, para todo $n \in \mathbb{Z}$, tenemos

$$
f(x) \in W_{n+1} \Leftrightarrow f\left(2^{n+1} x\right) \in V_{-1}-V_{0}=W_{0}
$$

ó

$$
f(x) \in W_{0} \Leftrightarrow f\left(2^{-n} x\right) \in W_{n} .
$$

Lema 2.1. Sea $\phi \in L^{2}(\mathbb{R})$. Entonces

$$
\{\phi(x-n)\}_{n \in \mathbb{Z}} \text { es una b.o.n para } L^{2}(\mathbb{R}) \Leftrightarrow \sum_{n \in \mathbb{Z}}|\widehat{\phi}(\omega+n)|^{2}=1 \text {, en c.t.p }
$$

Prueba. Sea $\phi_{0, n}(x)=\phi(x-n)$, entonces $\widehat{\phi}_{0, n}(\omega)=e^{2 \pi i \omega n} \widehat{\phi}(\omega)$. Consideremos $n=m-\ell$, entonces tomando $\ell=0$,

$$
\begin{aligned}
\delta_{0, n} & =\left\langle\widehat{\phi}_{0,0}, \widehat{\phi}_{0, n}\right\rangle=\left\langle\widehat{\phi}, \widehat{\phi}_{0, n}\right\rangle=\int_{-\infty}^{\infty} \widehat{\phi}(\omega) \overline{\widehat{\phi}}_{0, n}(\omega) d \omega \\
& =\int_{-\infty}^{\infty} \widehat{\phi}(\omega) e^{-2 \pi i \omega n} \overline{\widehat{\phi}}(\omega) d \omega=\int_{-\infty}^{\infty} e^{-2 \pi i \omega n}|\widehat{\phi}(\omega)|^{2} d \omega \\
& =\sum_{k \in \mathbb{Z}} \int_{k}^{k+1} e^{-2 \pi i \omega n}|\widehat{\phi}(\omega)|^{2} d \omega=\int_{0}^{1} e^{-2 \pi i \omega n} \sum_{k \in \mathbb{Z}}|\widehat{\phi}(\omega+k)|^{2} d \omega
\end{aligned}
$$


esto es,

$$
\delta_{0, n}=\left\langle\phi_{0,0}, \phi_{0, n}\right\rangle \Leftrightarrow \sum_{k \in \mathbb{Z}}|\widehat{\phi}(\omega+k)|^{2}=1 \text {, en c.t.p. }
$$

Objetivo: Construir una base ortonormal de ondículas para $L^{2}(\mathbb{R})$. Esto es, existe $\Psi \in W_{0}$ tal que

$$
\left\{\Psi_{m, n}=2^{-\frac{m}{2}} \Psi\left(2^{-m} x-n\right)\right\}_{m, n \in \mathbb{Z}}
$$

es una base ortonormal para $L^{2}(\mathbb{R})$. Para ello es suficiente mostrar que $\left\{\Psi_{0, n}\right\}$ es una base ortonormal para $W_{0}$.

Lema 2.2. La función escala $\phi$ de un AMR satisface:

i) $\sum_{k \in \mathbb{Z}}|\widehat{\phi}(\omega+k)|^{2}=1$, en c.t.p.

ii) $\widehat{\phi}(\omega)=M\left(\frac{\omega}{2}\right) \widehat{\phi}\left(\frac{\omega}{2}\right)$, donde $M(\omega)$ es una función periódica con periodo $1, M \in$ $L^{2}([0,1])$. Además:

$$
|M(\omega)|^{2}+\left|M\left(\omega+\frac{1}{2}\right)\right|^{2}=1, \text { en c.t.p. }
$$

\section{Prueba.}

i) Inmediato. Debido a la condición $v$ ) de la Definición del $A M R$ y el Lema 2.2.

ii) Veamos, como $\phi \in V_{0} \subset V_{-1}$ y $\left\{\phi_{-1, n}(x)=2^{1 / 2} \phi(2 x-n)\right\}_{n \in \mathbb{Z}}$ es una b.o.n. para $V_{-1}$, entonces

$$
\phi(x)=\sum_{n \in \mathbb{Z}} 2^{1 / 2}\left\langle\phi, \phi_{-1, n}\right\rangle \phi(2 x-n)
$$

donde

$$
\sum_{n \in \mathbb{Z}}\left|\alpha_{n}\right|^{2}<\infty, \alpha_{n}=\left\langle\phi, \phi_{-1, n}\right\rangle
$$

Tomando transformada de Fourier, tenemos

$$
\begin{aligned}
\widehat{\phi}(\omega) & =\sum_{n \in \mathbb{Z}} 2^{-1 / 2} \alpha_{n} e^{2 \pi i \omega n} \widehat{\phi}\left(\frac{\omega}{2}\right)=M\left(\frac{\omega}{2}\right) \widehat{\phi}\left(\frac{\omega}{2}\right), \\
M(\omega) & =2^{-1 / 2} \sum_{n \in \mathbb{Z}} \alpha_{n} e^{2 \pi i \omega n}
\end{aligned}
$$

Afirmación: $M(\omega+1)=M(\omega) ; M(\omega) \in L^{2}([0,1])$.

En efecto,

$$
\begin{aligned}
M(\omega+1) & =\sum_{n} 2^{-1 / 2} \alpha_{n} e^{2 \pi i(\omega+1) n}=\sum_{n} 2^{-1 / 2} \alpha_{n} e^{2 \pi i \omega n} e^{2 \pi i n} \\
& =\sum_{n \in \mathbb{Z}} 2^{-1 / 2} \alpha_{n} e^{2 \pi i \omega n}=M(\omega) .
\end{aligned}
$$


Asimismo,

$$
\begin{aligned}
\langle M(\omega), M(\omega)\rangle & =\left\langle\sum_{n} 2^{-1 / 2} \alpha_{n} e^{2 \pi i \omega n}, \sum_{k} 2^{-1 / 2} \alpha_{k} e^{2 \pi i \omega k}\right\rangle \\
& =\sum_{n} \sum_{m} 2^{-1} \alpha_{n} \bar{\alpha}_{k}\left\langle e^{2 \pi i \omega n}, e^{2 \pi i \omega k}\right\rangle \\
& =2^{-1} \sum_{n} \alpha_{n} \bar{\alpha}_{n}=2^{-1} \sum_{n}\left|\alpha_{n}\right|^{2}<\infty
\end{aligned}
$$

Por tanto $M(\omega) \in L^{2}([0,1])$.

Finalmente,

$$
\begin{aligned}
1= & \sum_{k}|\widehat{\phi}(\omega+k)|^{2}=\sum_{k}\left|M\left(\frac{\omega+k}{2}\right)\right|^{2}\left|\widehat{\phi}\left(\frac{\omega+k}{2}\right)\right|^{2} \\
1= & \sum_{k=2 m}\left|M\left(\frac{\omega}{2}+m\right)\right|^{2}\left|\widehat{\phi}\left(\frac{\omega}{2}+m\right)\right|^{2} \\
& +\sum_{k=2 m+1}\left|M\left(\frac{\omega}{2}+m+\frac{1}{2}\right)\right|^{2}\left|\widehat{\phi}\left(\frac{\omega}{2}+m+\frac{1}{2}\right)\right|^{2},
\end{aligned}
$$

pero, debido a que $M(\omega)$ es una función periódica, tenemos que

$$
M\left(\frac{\omega}{2}+m\right)=M\left(\frac{\omega}{2}\right), M\left(\frac{\omega}{2}+m+\frac{1}{2}\right)=M\left(\frac{\omega}{2}+\frac{1}{2}\right),
$$

entonces

$$
\begin{aligned}
& 1=\left|M\left(\frac{\omega}{2}\right)\right|^{2} \sum_{k=2 m}\left|\widehat{\phi}\left(\frac{\omega}{2}+m\right)\right|^{2}+\sum_{k=2 m+1}\left|M\left(\frac{\omega}{2}+\frac{1}{2}+m\right)\right|^{2}\left|\widehat{\phi}\left(\frac{\omega}{2}+\frac{1}{2}+m\right)\right|^{2} \\
& 1=\left|M\left(\frac{\omega}{2}\right)\right|^{2} \sum_{k=2 m}\left|\widehat{\phi}\left(\frac{\omega}{2}+m\right)\right|^{2}+\left|M\left(\frac{\omega}{2}+\frac{1}{2}\right)\right|^{2} \sum_{k=2 m+1}\left|\widehat{\phi}\left(\frac{\omega}{2}+\frac{1}{2}+m\right)\right|^{2}
\end{aligned}
$$

Por lo tanto

$$
\left|M\left(\frac{\omega}{2}\right)\right|^{2}+\left|M\left(\frac{\omega}{2}+\frac{1}{2}\right)\right|^{2}=1, \text { en c.t.p., }
$$

debido a que

$$
\sum_{k=2 m}\left|\widehat{\phi}\left(\frac{\omega}{2}+m\right)\right|^{2}=1, \sum_{k=2 m+1}\left|\widehat{\phi}\left(\frac{\omega}{2}+\frac{1}{2}+m\right)\right|^{2}=1 .
$$

Lema 2.3. Sea $f \in W_{0}$ entonces $\widehat{f}(\omega)=\lambda_{f}(\omega) \widehat{\Psi}(\omega)$, donde $\lambda_{f}$ es una funció $n$ periódica con periodo 1 y $\Psi$ es independiente de $\widehat{f}$. Además, $\lambda_{f} \in L^{2}([0,1]) y$

$$
\|f\|_{L^{2}}=\left\|\lambda_{f}\right\|_{L^{2}(I)}, \text { donde } I=[0,1] .
$$

Prueba. Sea $f \in W_{0}=V_{-1}-V_{0}$. Por consiguiente, $f \in V_{-1}$ y $f \perp V_{0}$, entonces

$$
f(x)=\sqrt{2} \sum_{n \in \mathbb{Z}} f_{n} \phi(2 x-n)
$$


donde $f_{n}=\left\langle f, \phi_{-1, n}\right\rangle$ y $\sum_{n \in \mathbb{Z}}\left|f_{n}\right|^{2}<\infty$.

Asimismo, repitiendo los argumentos usados para obtener el lema $2.3 \mathrm{ii}$ ), se tiene

$$
\widehat{f}(\omega)=M_{f}\left(\frac{\omega}{2}\right) \widehat{\phi}\left(\frac{\omega}{2}\right)
$$

donde $M_{f}(\omega)=\frac{1}{\sqrt{2}} \sum_{n} f_{n} e^{2 \pi i \omega n}$ y $M_{f}$ es una función periódica de periódo 1 ,

$M_{f} \in L^{2}([0,1])$. Como $f \perp V_{0}$, entonces

$$
\begin{aligned}
0 & =\left\langle f, \phi_{0, n}\right\rangle=\left\langle\widehat{f}, \widehat{\phi}_{0, n}\right\rangle=\int_{\mathbb{R}} \widehat{f}(\omega) \overline{\hat{\phi}}_{0, n}(\omega) d \omega=\int_{\mathbb{R}} \widehat{f}(\omega) \overline{e^{2 \pi i \omega n} \widehat{\phi}(\omega)} d \omega \\
& =\int_{\mathbb{R}} \widehat{f}(\omega) \overline{\widehat{\phi}}(\omega) e^{-2 \pi i \omega n} d \omega=\int_{0}^{1} e^{-2 \pi i \omega n}\left(\sum_{k \in \mathbb{Z}} \widehat{f}(\omega+k) \overline{\widehat{\phi}}(\omega+k)\right) d \omega
\end{aligned}
$$

esto es,

$$
\sum_{k \in \mathbb{Z}} \widehat{f}(\omega+k) \overline{\widehat{\phi}}(\omega+k)=0, \text { en c.t.p. }
$$

donde las series convergen absolutamente y en $L^{1}([0,1])$ debido a que $\widehat{f}, \widehat{\phi} \in L^{2}(\mathbb{R})$.

Usando $\widehat{\phi}(\omega)=M\left(\frac{\omega}{2}\right) \widehat{\phi}\left(\frac{\omega}{2}\right)$ y $(2.4 .1)$ en (2.4.2), obtenemos

$$
\sum_{k \in \mathbb{Z}} M_{f}\left(\frac{\omega+k}{2}\right) \hat{\phi}\left(\frac{\omega+k}{2}\right)\left[\overline{M\left(\frac{\omega+k}{2}\right) \widehat{\phi}\left(\frac{\omega+k}{2}\right)}\right]=0 \text {, en c.t.p., }
$$

descomponiendo la serie en $k=2 n$ y $k=2 n+1$, y usando

$$
\sum_{k \in \mathbb{Z}}|\widehat{\phi}(\omega+k)|^{2}=1, \text { en c.t.p. }
$$

obtenemos

$$
M_{f}(\omega) \overline{M(\omega)}+M_{f}\left(\omega+\frac{1}{2}\right) \overline{M\left(\omega+\frac{1}{2}\right)}=0, \text { en c.t.p. }
$$

Asimismo como

$$
|M(\omega)|^{2}+\left|M\left(\omega+\frac{1}{2}\right)\right|^{2}=1, \text { en c.t.p. }
$$

esto significa que $M(\omega)$ y $M\left(\omega+\frac{1}{2}\right)$ no pueden anularse simultanéamente sobre un conjunto de medida positiva, entonces podemos decir

$$
M_{f}(\omega)=\frac{-M_{f}\left(\omega+\frac{1}{2}\right)}{\overline{M(\omega)}} \cdot \overline{M\left(\omega+\frac{1}{2}\right)} \Leftrightarrow M_{f}(\omega)=K_{f}(\omega) \cdot \overline{M\left(\omega+\frac{1}{2}\right)} \text {, en c.t.p. }
$$

donde $K_{f}(\omega)=\frac{-M_{f}\left(\omega+\frac{1}{2}\right)}{\overline{M(\omega)}}$, entonces 


$$
\begin{aligned}
K_{f}(\omega)+K_{f}\left(\omega+\frac{1}{2}\right) & =\frac{-M_{f}\left(\omega+\frac{1}{2}\right)}{\overline{M(\omega)}}+\frac{-M_{f}(\omega+1)}{\overline{M\left(\omega+\frac{1}{2}\right)}} \\
& =-\left(\frac{M_{f}\left(\omega+\frac{1}{2}\right) \overline{M\left(\omega+\frac{1}{2}\right)}+M_{f}(\omega+1) \overline{M(\omega)}}{\overline{M(\omega)} M\left(\omega+\frac{1}{2}\right)}\right) \\
& =0 \text {, en c.t.p. }
\end{aligned}
$$

(debido a que

$$
\left.M_{f}(\omega) \overline{M(\omega)}+M_{f}\left(\omega+\frac{1}{2}\right) \overline{M\left(\omega+\frac{1}{2}\right)}=0, \text { en c.t.p. }\right)
$$

Por lo tanto, podemos asumir que $K_{f}(\omega)=e^{2 \pi i \omega} \lambda_{f}(2 \omega)$, donde $\lambda_{f}(2 \omega)$ es pe-riódica de periodo 1 , esto es, $\lambda_{f}(2 \omega+1)=\lambda_{f}(2 \omega)$. De este modo,

$$
M_{f}(\omega)=K_{f}(\omega) \overline{M\left(\omega+\frac{1}{2}\right)}=e^{2 \pi i \omega} \lambda_{f}(2 \omega) \overline{M\left(\omega+\frac{1}{2}\right)}
$$

Pero, por (2.4.1) tenemos

$$
\widehat{f}(\omega)=e^{\pi i \omega} M\left(\frac{\omega}{2}+\frac{1}{2}\right) \widehat{\phi}\left(\frac{\omega}{2}\right) \lambda_{f}(\omega)
$$

Por consiguiente

$$
\widehat{f}(\omega)=\widehat{\Psi}(\omega) \lambda_{f}(\omega)
$$

donde

$$
\widehat{\Psi}(\omega)=e^{\pi i \omega} M\left(\frac{\omega}{2}+\frac{1}{2}\right) \widehat{\phi}\left(\frac{\omega}{2}\right)
$$

Finalmente,

$$
\begin{aligned}
\left\|M_{f}\right\|_{L^{2}(I)}^{2}= & \int_{0}^{1}\left|M_{f}(\omega)\right|^{2} d \omega=\int_{0}^{1}\left|e^{2 \pi i \omega}\right|^{2}\left|\lambda_{f}(2 \omega)\right|^{2}\left|\overline{M\left(\omega+\frac{1}{2}\right)}\right|^{2} d \omega \\
\left\|M_{f}\right\|_{L^{2}(I)}^{2}= & \left.\int_{0}^{1}\left|\lambda_{f}(2 \omega)\right|^{2} \overline{M_{f}\left(\omega+\frac{1}{2}\right)}\right|^{2} d \omega=\int_{0}^{1 / 2}\left|\lambda_{f}(2 \omega)\right|^{2}\left|\overline{M\left(\omega+\frac{1}{2}\right)}\right|^{2} d \omega \\
& +\int_{1 / 2}^{1}\left|\lambda_{f}(2 \omega)\right|^{2}\left|\overline{M\left(\omega+\frac{1}{2}\right)}\right|^{2} d \omega
\end{aligned}
$$

pero, debido a que $\lambda_{f}, M(\omega)$ son funciones periódicas de periodo 1 , entonces 


$$
\begin{aligned}
\int_{1 / 2}^{1}\left|\lambda_{f}(2 \omega)\right|^{2}\left|\overline{M\left(\omega+\frac{1}{2}\right)}\right|^{2} d \omega & =\int_{0}^{1 / 2}\left|\lambda_{f}\left[2\left(\omega+\frac{1}{2}\right)\right]\right|^{2}\left|\overline{\mid M\left(\frac{1}{2}+\frac{1}{2}\right)}\right|^{2} d \omega \\
& =\int_{0}^{1 / 2}\left|\lambda_{f}(2 \omega+1)\right|^{2}|M(\omega+1)|^{2} d \omega \\
& =\int_{0}^{1 / 2}\left|\lambda_{f}(2 \omega)\right|^{2}|\overline{M(\omega)}|^{2} d \omega
\end{aligned}
$$

Por consiguiente

$$
\left\|M_{f}\right\|_{L^{2}(I)}^{2}=\int_{0}^{1 / 2}\left|\lambda_{f}(2 \omega)\right|^{2}\left[|M(\omega)|^{2}+\left|M\left(\omega+\frac{1}{2}\right)\right|^{2}\right] d \omega
$$

Luego, debido a que $|M(\omega)|^{2}+\left|M\left(\omega+\frac{1}{2}\right)\right|^{2}=1$, en c.t.p y haciendo $2 \omega=\mu$, obtenemos

$$
\left\|M_{f}\right\|_{L^{2}(I)}^{2}=\frac{1}{2} \int_{0}^{1}\left|\lambda_{f}(2 \mu)\right|^{2} d \mu
$$

es decir,

$$
\left\|M_{f}\right\|_{L^{2}(I)}^{2}=\frac{1}{2} \int_{0}^{1}\left|\lambda_{f}(\omega)\right|^{2} d \omega .
$$

y debido a que $M_{f} \in L^{2}(I)$, se tiene que $\lambda_{f} \in L^{2}(I)$.

Asimismo

$$
\begin{aligned}
\left\|M_{f}\right\|_{L^{2}(I)}^{2} & =\left\langle\frac{1}{\sqrt{2}} \sum_{m} f_{m} e^{2 \pi i m \omega}, \frac{1}{\sqrt{2}} \sum_{n} f_{n} e^{2 \pi i n \omega}\right\rangle \\
& =\frac{1}{2} \sum_{m} \sum_{n} f_{m} f_{n}\left\langle e^{2 \pi i m \omega}, e^{2 \pi i n \omega}\right\rangle \\
& =\frac{1}{2} \sum_{n}\left|f_{n}\right|^{2}=\frac{1}{2}\|f\|_{L^{2}}^{2},
\end{aligned}
$$

donde $f_{n}=\left\langle f, \phi_{-1, n}\right\rangle$. Por consiguiente

$$
|f|_{L^{2}(I)}=\left|\lambda_{f}\right|_{L^{2}(I)}
$$

Teorema 2.1. Dado un $A M R$ para $L^{2}(\mathbb{R})$, existe una b.o.n de Ondículas,

$$
\left\{\Psi_{m, n}(x)=2^{-m / 2} \Psi\left(2^{-m} x-n\right)\right\}_{m, n \in \mathbb{Z}} \text { para } L^{2}(\mathbb{R}) .
$$

Prueba. Es suficiente mostrar que existe $\Psi \in W_{0}$ tal que $\{\Psi(x-n)\}_{n \in \mathbb{Z}}$ es una b.o.n para $W_{0}$. Consideremos $\Psi$ como anteriormente, esto es,

$$
\widehat{\Psi}(\omega)=e^{\pi i \omega} \overline{M\left(\frac{\omega}{2}+\frac{1}{2}\right)} \widehat{\phi}\left(\frac{\omega}{2}\right) .
$$

Sea $f \in W_{0}$, entonces es conocido que $\widehat{f}(\omega)=\lambda_{f}(\omega) \widehat{\Psi}(\omega)$, donde $\lambda_{f}$ es una función periódica de periodo $1, \lambda_{f} \in L^{2}(I)$, la cual puede escribirse como 


$$
\lambda_{f}(\omega)=\sum_{n \in \mathbb{Z}} \hat{\lambda}_{f}(n) e^{2 \pi i n \omega}
$$

$\operatorname{con} \hat{\lambda}_{f}(n)=\left\langle\lambda_{f}(\omega), e^{2 \pi i n \omega}\right\rangle, \mathrm{y}\left\|\lambda_{f}\right\|_{L^{2}(I)}^{2}=\sum_{n \in \mathbb{Z}}\left|\widehat{\lambda}_{f}(n)\right|^{2}<\infty$.

Reemplazando $\lambda_{f}(\omega)$ en $\widehat{f}(\omega)$, se tiene

$$
\widehat{f}(\omega)=\sum_{n \in \mathbb{Z}} \widehat{\lambda}_{f}(n) e^{2 \pi i n \omega} \widehat{\Psi}(\omega)=\sum_{n \in \mathbb{Z}} \widehat{\lambda}_{f}(n)[\Psi(x-n) \hat{]}(\omega),
$$

donde $\left[\Psi(x-n) \hat{]}(\omega)=e^{2 \pi i n \omega} \widehat{\Psi}(\omega)\right.$ y tomando la anti-transformada de Fourier, obtenemos

$$
f(x)=\sum_{n \in \mathbb{Z}} \widehat{\lambda}_{f}(n) \Psi(x-n)
$$

Para ver que $\{\Psi(x-n)\}_{n \in \mathbb{Z}}$ es un b.o.n. para $W_{0}$, necesitamos verificar que:

i) $\Psi \in W_{0}$

ii) $\{\Psi(x-n)\}_{n \in \mathbb{Z}}$ es ortonormal

iii) (2.5.1) converge en $L^{2}(\mathbb{R})$ y $\|f\|_{L^{2}(\mathbb{R})}=\sum_{n \in \mathbb{Z}}\left|\widehat{\lambda}_{f}(n)\right|^{2}$.

A continuación probemos las condiciones i), ii) y iii).

Prueba de iii). En efecto, debido a

$$
\|f\|_{L^{2}(\mathbb{R})}=\left\|\lambda_{f}\right\|_{L^{2}(I)}=\left\|\widehat{\lambda}_{f}\right\|_{\ell^{2}(\mathbb{Z})}=\sum_{n \in \mathbb{Z}}\left|\widehat{\lambda}_{f}(n)\right|^{2}<\infty .
$$

Prueba de ii). Como $\{\Psi(x-n)\}_{n \in \mathbb{Z}}$ es b.o.n., entonces es equivalente a tener

$$
\sum_{n \in \mathbb{Z}}|\widehat{\Psi}(\omega+n)|^{2}=1 \text {, en c.t.p. }
$$

En efecto, debido a que $M(\omega)$ es periódica de periodo 1 y $\sum_{k}|\widehat{\phi}(\omega+k)|^{2}=1$, en c.t.p., se tiene

$$
\begin{aligned}
\sum_{n}|\widehat{\Psi}(\omega+n)|^{2}= & \sum_{n}\left|e^{\pi i \omega}\right|^{2}\left|M\left(\frac{\omega}{2}+\frac{n}{2}+\frac{1}{2}\right)\right|^{2}\left|\widehat{\phi}\left(\frac{\omega}{2}+\frac{n}{2}\right)\right|^{2} \\
\sum_{n}|\widehat{\Psi}(\omega+n)|^{2}= & \sum_{n=2 k}\left|M\left(\frac{\omega}{2}+k+\frac{1}{2}\right)\right|^{2}\left|\widehat{\phi}\left(\frac{\omega}{2}+k\right)\right|^{2} \\
& +\sum_{n=2 k+1}\left|M\left(\frac{\omega}{2}+k+1\right)\right|^{2}\left|\widehat{\phi}\left(\frac{\omega}{2}+k+\frac{1}{2}\right)\right|^{2} \\
= & \left|M\left(\frac{\omega}{2}+\frac{1}{2}\right)\right|^{2} \sum_{k}\left|\widehat{\phi}\left(\frac{\omega}{2}+k\right)\right|^{2} \\
& +\left|M\left(\frac{\omega}{2}\right)\right|^{2} \sum_{k}\left|\widehat{\phi}\left(\frac{\omega}{2}+k+\frac{1}{2}\right)\right|^{2} \\
= & \left|M\left(\frac{\omega}{2}+\frac{1}{2}\right)\right|^{2}+\left|M\left(\frac{\omega}{2}\right)\right|^{2}=1, \text { en c.t.p. },
\end{aligned}
$$


Asimismo,

$$
\left\langle\Psi_{0,0} \Psi_{0, n}\right\rangle=\left\langle\Psi, \Psi_{0, n}\right\rangle=\int_{0}^{1} e^{-2 \pi i \omega n} d \omega=\delta_{0, n}
$$

En efecto, debido a que $\left[g(x-n) \hat{]}(\omega)=e^{2 \pi i \omega n} \widehat{g}(\omega)\right.$, se tiene

$$
\begin{aligned}
\left\langle\Psi, \Psi_{0, n}\right\rangle & =\left\langle\widehat{\Psi}, \widehat{\Psi}_{0, n}\right\rangle=\int_{-\infty}^{\infty} \widehat{\Psi}(\omega) \overline{[\Psi(x-n) \overline{]}}(\omega) d \omega \\
& =\int_{-\infty}^{\infty} \widehat{\Psi}(\omega) e^{-2 \pi i \omega n} \widehat{\widehat{\Psi}}(\omega) d \omega=\int_{-\infty}^{\infty}|\widehat{\Psi}(\omega)|^{2} e^{-2 \pi i \omega n} d \omega
\end{aligned}
$$

Pero,

$$
\begin{aligned}
\int_{1}^{2}|\widehat{\Psi}(\omega)|^{2} e^{-2 \pi i \omega n} d \omega & =\int_{1-1}^{2-1}|\widehat{\Psi}(\omega+1)|^{2} e^{-2 \pi i(\omega+1) n} d \omega=\int_{0}^{1}|\widehat{\Psi}(\omega+1)|^{2} e^{-2 \pi i \omega n} d \omega \\
\int_{2}^{3}|\widehat{\Psi}(\omega)|^{2} e^{-2 \pi i \omega n} d \omega & =\int_{2-2}^{3-2}|\widehat{\Psi}(\omega+2)|^{2} e^{-2 \pi i \omega n} d \omega=\int_{0}^{1}|\widehat{\Psi}(\omega+2)|^{2} e^{-2 \pi i \omega n} d \omega \\
& \vdots \\
\int_{-1}^{0}|\widehat{\Psi}(\omega)|^{2} e^{-2 \pi i \omega n} d \omega & =\int_{-1+1}^{0+1}|\widehat{\Psi}(\omega-1)|^{2} e^{-2 \pi i \omega n} d \omega=\int_{0}^{1}|\widehat{\Psi}(\omega-1)|^{2} e^{-2 \pi i \omega n} d \omega \\
\int_{-2}^{-1}|\widehat{\Psi}(\omega)|^{2} e^{-2 \pi i \omega n} d \omega & =\int_{-2+2}^{-1+1}|\widehat{\Psi}(\omega-2)|^{2} e^{-2 \pi i \omega n} d \omega=\int_{0}^{1}|\widehat{\Psi}(\omega-2)|^{2} e^{-2 \pi i \omega n} d \omega
\end{aligned}
$$

entonces

$$
\begin{aligned}
& \int_{-\infty}^{\infty}|\widehat{\Psi}(\omega)|^{2} e^{-2 \pi i \omega n} d \omega=\cdots+\int_{-2}^{-1}|\widehat{\Psi}(\omega)|^{2} e^{-2 \pi i \omega n} d \omega+\int_{-1}^{0}|\widehat{\Psi}(\omega)|^{2} e^{-2 \pi i \omega n} d \omega \\
& +\int_{0}^{1}|\widehat{\Psi}(\omega)|^{2} e^{-2 \pi i \omega n} d \omega+\int_{1}^{2}|\widehat{\Psi}(\omega)|^{2} e^{-2 \pi i \omega n} d \omega+\int_{2}^{3}|\widehat{\Psi}(\omega)|^{2} e^{-2 \pi i \omega n} d \omega+\cdots
\end{aligned}
$$

es decir

$$
\begin{aligned}
\int_{-\infty}^{\infty}|\widehat{\Psi}(\omega)|^{2} e^{-2 \pi i \omega n} d \omega & =\int_{0}^{1} \sum_{k \in \mathbb{Z}}|\widehat{\Psi}(\omega+k)|^{2} e^{-2 \pi i \omega n} d \omega \\
& =\int_{0}^{1} e^{-2 \pi i \omega n} d \omega=\delta_{0, n}
\end{aligned}
$$

(debido a que $\sum_{k \in \mathbb{Z}}|\widehat{\Psi}(\omega+k)|^{2}=1$ en c.t.p.)

Prueba de i). Basta probar lo siguiente:

a) $\Psi \in V_{-1}$

b) $\Psi \perp V_{0}$

a) En efecto, como $\widehat{\Psi}(\omega)=e^{\pi i \omega} M\left(\frac{\omega}{2}+\frac{1}{2}\right) \widehat{\phi}\left(\frac{\omega}{2}\right)$ entonces $\widehat{\Psi}$ podemos escribirla 
en la forma

$$
\widehat{\Psi}(\omega)=G(\omega) \widehat{\phi}\left(\frac{\omega}{2}\right)
$$

donde $G(\omega)=e^{\pi i \omega} \overline{M\left(\frac{\omega}{2}+\frac{1}{2}\right)}$ es una función periódica en $L^{2}([0,2])$ con periódo $2(G(\omega+2)=$ $G(\omega))$.

Por consiguiente podemos escribir

$$
G(\omega)=2^{-\frac{1}{2}} \sum_{n \in \mathbb{Z}} \widetilde{g}_{n} e^{\pi i n \omega} \widehat{\phi}\left(\frac{\omega}{2}\right)
$$

con $\|G\|_{L^{2}}=\sum_{n}\left|\widetilde{g}_{n}\right|^{2}<\infty$, reemplazando $G(\omega)$ en $\widehat{\Psi}(\omega)$ obtenemos

$$
\widehat{\Psi}(\omega)=\sum_{n \in \mathbb{Z}} 2^{-\frac{1}{2}} \widetilde{g}_{n} e^{\pi i n \omega} \widehat{\phi}\left(\frac{\omega}{2}\right)
$$

y tomando anti-tranformada de Fourier, se obtiene

$$
[\widehat{\Psi}(\omega)]^{\vee}(x)=\sum_{n \in \mathbb{Z}} \widetilde{g}_{n}\left(2^{-\frac{1}{2}} e^{\pi i n \omega} \widehat{\phi}\left(\frac{\omega}{2}\right)\right)^{\vee}=2^{\frac{1}{2}} \sum_{n \in \mathbb{Z}} \widetilde{g}_{n} \phi(2 x-n) .
$$

Luego

$$
\Psi(x)=2^{\frac{1}{2}} \sum_{n \in \mathbb{Z}} \widetilde{g}_{n} \phi(2 x-n),\{\phi(2 x-n)\}_{n \in \mathbb{Z}} \text { es b.o.n. para } V_{-1} .
$$

Por lo tanto $\Psi \in V_{-1}$.

b) Es suficiente probar que $\left\langle\Psi, \phi_{0, n}\right\rangle=0 \forall n \in \mathbb{Z}$, donde $\phi_{0, n}(x)=\phi(x-n)$.

En efecto,

$$
\begin{aligned}
\left\langle\Psi, \phi_{0, n}\right\rangle & =\left\langle\widehat{\Psi}, \widehat{\phi}_{0, n}\right\rangle=\int_{-\infty}^{\infty} \widehat{\Psi}(\omega) \overline{\widehat{\phi}}_{0, n}(\omega) d \omega=\int_{-\infty}^{\infty} \widehat{\Psi}(\omega) \overline{e^{2 \pi i n \omega} \widehat{\phi}(\omega)} d \omega \\
& =\int_{-\infty}^{\infty} \widehat{\Psi}(\omega) e^{-2 \pi i n \omega} \overline{\widehat{\phi}}(\omega) d \omega \\
& =\int_{0}^{1} e^{-2 \pi i n \omega}\left(\sum_{k \in \mathbb{Z}} \widehat{\Psi}(\omega+k) \overline{\widehat{\phi}(\omega+k)}\right) d \omega
\end{aligned}
$$

Pero,

$$
\begin{aligned}
& \sum_{k \in \mathbb{Z}} \widehat{\Psi}(\omega+k) \overline{\widehat{\phi}(\omega+k)}=\sum_{k \in \mathbb{Z}} e^{\pi i(\omega+k)} \overline{M\left(\frac{\omega+k}{2}+\frac{1}{2}\right)} \widehat{\phi}\left(\frac{\omega+k}{2}\right) \overline{M\left(\frac{\omega+k}{2}\right) \widehat{\phi}\left(\frac{\omega+k}{2}\right)} \\
& \text { (Debido a que } \widehat{\Psi}(\omega)=e^{\pi i \omega} \overline{M\left(\frac{\omega}{2}+\frac{1}{2}\right)} \widehat{\phi}\left(\frac{\omega}{2}\right) \text { y } \widehat{\phi}(\omega)=M\left(\frac{\omega}{2}\right) \widehat{\phi}\left(\frac{\omega}{2}\right) \text {.) }
\end{aligned}
$$




$$
\begin{aligned}
= & \sum_{k \in \mathbb{Z}} e^{\pi i \omega} \cdot e^{\pi i k} \overline{M\left(\frac{\omega}{2}+\frac{k}{2}+\frac{1}{2}\right)} \overline{M\left(\frac{\omega}{2}+\frac{k}{2}\right)}\left|\widehat{\phi}\left(\frac{\omega}{2}+\frac{k}{2}\right)\right|^{2} \\
= & \sum_{k=2 m} e^{\pi i \omega} M \overline{\left(\frac{\omega}{2}+m+\frac{1}{2}\right)} \overline{M\left(\frac{\omega}{2}+m\right)}\left|\hat{\phi}\left(\frac{\omega}{2}+m\right)\right|^{2} \\
& -\sum_{k=2 m+1} e^{\pi i \omega} \overline{M\left(\frac{\omega}{2}+m+1\right)} \overline{M\left(\frac{\omega}{2}+m+\frac{1}{2}\right)}\left|\widehat{\phi}\left(\frac{\omega}{2}+m+\frac{1}{2}\right)\right|^{2} \\
= & e^{\pi i \omega} \overline{M\left(\frac{\omega}{2}\right)} \overline{M\left(\frac{\omega}{2}+\frac{1}{2}\right)}\left(\sum_{m}\left|\widehat{\phi}\left(\frac{\omega}{2}+m\right)\right|^{2}-\sum_{m}\left|\widehat{\phi}\left(\frac{\omega}{2}+m+\frac{1}{2}\right)\right|^{2}\right) \\
= & 0 .
\end{aligned}
$$

(Hemos usado que $\sum_{k}|\widehat{\phi}(\omega+k)|^{2}=1$, en c.t.p. y que $M(\omega)$ es una función periódica de periodo 1.)

Asi, hemos probado que

\section{Corolario 3.}

$$
\left\langle\Psi, \phi_{0, n}\right\rangle=0, \forall n
$$

La ondícula madre $\Psi$ puede ser explicitada vía

$$
\Psi(x)=\sum_{n \in \mathbb{Z}}(-1)^{1-n} \alpha_{1-n} \phi_{-1, n}(x),
$$

donde los coeficientes $\alpha_{n}$ estan dados por

$$
\phi(x)=\sum_{n \in \mathbb{Z}} \alpha_{n} \phi_{-1, n}(x),
$$

$\operatorname{con} \alpha_{n}=\left\langle\phi, \phi_{-1, n}\right\rangle$ y $\sum_{n \in \mathbb{Z}}\left|\alpha_{n}\right|^{2}<\infty$.

Prueba. En efecto, como

$$
M(\omega)=2^{-1 / 2} \sum_{n} \alpha_{n} e^{2 \pi i n \omega} \text { y } \widehat{\Psi}(\omega)=e^{\pi i \omega} \overline{M\left(\frac{\omega}{2}+\frac{1}{2}\right)} \widehat{\phi}\left(\frac{\omega}{2}\right),
$$

entonces

$$
\begin{aligned}
\widehat{\Psi}(\omega) & =e^{\pi i \omega}\left(2^{-1 / 2} \sum_{n} \alpha_{n} e^{2 \pi i n\left(\frac{\omega}{2}+\frac{1}{2}\right)}\right) \widehat{\phi}\left(\frac{\omega}{2}\right) \\
& =e^{\pi i \omega}\left(2^{-1 / 2} \sum_{n} \alpha_{n} e^{-\pi i \omega n} e^{-\pi i n}\right) \widehat{\phi}\left(\frac{\omega}{2}\right) \\
& =2^{-1 / 2} \sum_{n} \alpha_{n} e^{\pi i \omega(1-n)} \cdot(-1)^{n} \widehat{\phi}\left(\frac{\omega}{2}\right) \\
& =2^{-1 / 2}\left(\sum_{m} \alpha_{m-1} \cdot e^{\pi i \omega m}(-1)^{1-m} \widehat{\phi}\left(\frac{\omega}{2}\right)\right) \\
\widehat{\Psi}(\omega) & =\sum_{m} \alpha_{1-m} \cdot e^{\pi i \omega m}(-1)^{1-m} 2^{-1 / 2} \widehat{\phi}\left(\frac{\omega}{2}\right),
\end{aligned}
$$


tomando anti-transformada de Fourier, se obtiene

$$
[\widehat{\Psi}(\omega)]^{\vee}(x)=\sum_{m} \alpha_{1-m}(-1)^{1-m} 2^{1 / 2} \phi(2 x-m),
$$

esto es, .

$$
\Psi(x)=2^{1 / 2} \sum_{m}(-1)^{1-m} \alpha_{1-m} \phi_{-1, m}
$$

\section{Conclusiones}

3.1 La "ondícula madre" $\Psi$ asociada con un $A M R$ dado no es única por ejemplo, podemos tomar

$$
K_{f}(\omega)=e^{2 \pi i \omega} e^{2 \pi i p(\omega)} \lambda_{f}(2 \omega),
$$

donde $p(\omega)$ es una función real-valorada con $p\left(\omega+\frac{1}{2}\right)=p(\omega)$. Entonces

$$
\begin{aligned}
M_{f}(\omega) & =K_{f}(\omega) \overline{M\left(\frac{\omega}{2}+\frac{1}{2}\right)}=e^{2 \pi i \omega} e^{2 \pi i p(\omega)} \lambda_{f}(2 \omega) \overline{M\left(\omega+\frac{1}{2}\right)} \\
\widehat{f}(\omega) & =M_{f}\left(\frac{\omega}{2}\right) \widehat{\phi}\left(\frac{\omega}{2}\right)=e^{2 \pi i \frac{\omega}{2} e^{2 \pi i p\left(\frac{\omega}{2}\right)} \lambda_{f}\left(2 \cdot \frac{\omega}{2}\right) \overline{M\left(\frac{\omega}{2}+\frac{1}{2}\right)}} \widehat{\phi}\left(\frac{\omega}{2}\right) \\
\widehat{f}(\omega) & =\left(e^{\pi i \omega} M \overline{\left(\frac{\omega}{2}+\frac{1}{2}\right)} \widehat{\phi}\left(\frac{\omega}{2}\right)\right) e^{2 \pi i p\left(\frac{\omega}{2}\right)} \lambda_{f}(\omega) \\
\widehat{f}(\omega) & =e^{2 \pi i p\left(\frac{\omega}{2}\right)} \widehat{\Psi}(\omega) \lambda_{f}(\omega),
\end{aligned}
$$

donde

$$
\widehat{\Psi}(\omega)=e^{\pi i \omega} \overline{M\left(\frac{\omega}{2}+\frac{1}{2}\right)} \widehat{\phi}\left(\frac{\omega}{2}\right),
$$

por lo tanto, esto nos da una nueva ondícula madre $\widetilde{\Psi}$ que satisface

$$
\widehat{\widetilde{\Psi}}(\omega)=e^{2 \pi i p\left(\frac{\omega}{2}\right)} \widehat{\Psi}(\omega) .
$$

3.2 En particular si $p(\omega)=\frac{p}{2}$, donde $p$ es un entero impar, se obtiene que:

$$
\begin{aligned}
& \widehat{\widetilde{\Psi}}(\omega)=e^{2 \pi i p\left(\frac{\omega}{2}\right)} \sum_{n} \alpha_{1-n}(-1)^{1-n} 2^{-1 / 2} e^{\pi i \omega n} \widehat{\phi}\left(\frac{\omega}{2}\right) \\
& \widehat{\widetilde{\Psi}}(\omega)=e^{\pi i p} \sum_{n} \alpha_{1-n}(-1) \cdot(-1)^{-n} \cdot 2^{-1 / 2} e^{\pi i \omega n} \widehat{\phi}\left(\frac{\omega}{2}\right) \\
& \widehat{\widetilde{\Psi}}(\omega)=\sum_{n} \alpha_{1-n}(-1)^{n} \cdot 2^{-1 / 2} e^{\pi i \omega n} \widehat{\phi}\left(\frac{\omega}{2}\right),
\end{aligned}
$$

tomando la anti-transformada de Fourier, se obtiene 


$$
\begin{aligned}
{[\widehat{\widetilde{\Psi}}(\omega)]^{\vee}(x) } & =\sum_{n} \alpha_{1-n}(-1)^{n} \phi_{-1, n}(x) \\
\widetilde{\Psi}(x) & =\sum_{n} \alpha_{1-n}(-1)^{n} \phi_{-1, n}(x) .
\end{aligned}
$$

3.3 El recíproco del Teorema 2.5 no es verdadero, en el sentido que $\exists$ una b.o.n. de ondículas para $L^{2}(\mathbb{R})$ que no es generado a partir de algún $A M R$ (debido a J.L Journe)

3.4 El corolario 2.6 nos da una fórmula para la reconstrucción de la "ondícula madre" $\Psi$; por consiguiente, construimos una b.o.n. de "ondículas" para $L^{2}(\mathbb{R})$. Complementando el procedimiento desarrollado en el corolario 2.6 resulta más facil si la series

$$
\phi(x)=\sum_{n \in \mathbb{Z}} \alpha_{n} \sqrt{2} \phi(2 x-n) \operatorname{con} \sum_{n \in \mathbb{Z}}\left|\alpha_{n}\right|^{20}<\infty \text { y } \alpha_{n}=\left\langle\phi, \phi_{-1, n}\right\rangle
$$

tienen un número finito de términos no ceros.

El asumir que $\left\{\phi_{0, n}(x)=\phi(x-n)\right\}_{n \in \mathbb{Z}}$ es una b.o.n. para $V_{0}$. Juega un rol vital en la construcción de una b.o.n. de "ondículas" para $L^{2}(\mathbb{R})$ en el Teorema 2.5. Si deseamos que $\left\{\phi_{0, n}(x)=\phi(x-n)\right\}_{n \in \mathbb{Z}}$ sea sólo una base de Riesz para $V_{0}$ y no necesariamente una b.o.n., podriamos construir una b.o.n. de "ondículas" para $L^{2}(\mathbb{R})$.

Definición 4.1. $\left\{\phi_{0, n}\right\}_{n \in \mathbb{Z}}$ es una base de Riesz para $V_{0}$ si y solo si

i) $V_{0}=\left\langle\left\{\phi_{0, n}\right\}\right\rangle$.

ii) $\forall\left\{c_{n}\right\}_{n \in \mathbb{Z}} \in \ell^{2}(\mathbb{Z}), A \sum_{n}\left|c_{n}\right|^{2} \leq\left\|\sum_{n} c_{n} \phi(\cdot-n)\right\|^{2} \leq B \sum_{n}\left|c_{n}\right|^{2}$

donde $A>0,0<B<\infty$ son independientes de los $\left\{c_{n}\right\}_{n \in \mathbb{Z}}$.

Sea $f \in V_{0}$ entonces $f(x)=\sum_{n} c_{n} \phi(x-n) \Rightarrow \widehat{f}(\omega)=\sum_{n} c_{n} e^{2 \pi i \omega n} \widehat{\phi}(\omega)$.

$$
\begin{aligned}
\|f\|_{L^{2}}^{2} & =\|\widehat{f}\|_{L^{2}}=\int_{-\infty}^{\infty}|\widehat{f}(\omega)|^{2} d \omega=\int_{-\infty}^{\infty}\left|\sum_{n} c_{n} e^{2 \pi i \omega n} \widehat{\phi}(\omega)\right|^{2} d \omega \\
& =\int_{-\infty}^{\infty}\left|\left(\sum_{n} c_{n} e^{2 \pi i \omega n}\right) \widehat{\phi}(\omega)\right|^{2} d \omega \\
& =\cdots+\int_{-2}^{-1}+\int_{-1}^{0}+\int_{0}^{1}+\int_{1}^{2}+\cdots
\end{aligned}
$$

pero, 


$$
\begin{aligned}
\int_{-2}^{-1}\left|\left(\sum_{n} c_{n} e^{2 \pi i \omega n}\right) \widehat{\phi}(\omega)\right|^{2} d \omega & =\int_{-2+2}^{-1+2}\left|\left(\sum_{n} c_{n} e^{2 \pi i(\omega-2) n}\right) \widehat{\phi}(\omega-2)\right|^{2} d \omega \\
& =\int_{0}^{1}\left|\left(\sum_{n} c_{n} e^{2 \pi i \omega n}\right) \widehat{\phi}(\omega-2)\right|^{2} d \omega \\
\int_{-1}^{0}\left|\left(\sum_{n} c_{n} e^{2 \pi i \omega n}\right) \widehat{\phi}(\omega)\right|^{2} d \omega & =\int_{-1+1}^{0+1}\left|\left(\sum_{n} c_{n} e^{2 \pi i(\omega-1) n}\right) \widehat{\phi}(\omega-1)\right|^{2} d \omega \\
& =\int_{0}^{1}\left|\left(\sum_{n} c_{n} e^{2 \pi i \omega n}\right) \widehat{\phi}(\omega-1)\right|^{2} d \omega \\
\int_{1}^{2}\left|\left(\sum_{n} c_{n} e^{2 \pi i \omega n}\right) \widehat{\phi}(\omega)\right|^{2} d \omega & =\int_{1-1}^{2-1}\left|\left(\sum_{n} c_{n} e^{2 \pi i(\omega+1) n}\right) \widehat{\phi}(\omega+1)\right|^{2} d \omega \\
& =\int_{0}^{1}\left|\left(\sum_{n} c_{n} e^{2 \pi i \omega n}\right) \widehat{\phi}(\omega+1)\right|^{2} d \omega
\end{aligned}
$$

asi sucesivamente, esto es,

$$
\begin{aligned}
\|f\|_{L^{2}}^{2}= & \cdots+\int_{0}^{1}\left|\sum_{n} c_{n} e^{2 \pi i \omega n}\right|^{2}|\widehat{\phi}(\omega-2)|^{2} d \omega+\int_{0}^{1}\left|\sum_{n} c_{n} e^{2 \pi i \omega n}\right|^{2}|\widehat{\phi}(\omega-1)| d \omega \\
& +\int_{0}^{1}\left|\sum_{n} c_{n} e^{2 \pi i \omega n}\right|^{2}|\widehat{\phi}(\omega)|^{2} d \omega+\int_{0}^{1}\left|\sum_{n} c_{n} e^{2 \pi i \omega n}\right|^{2}|\widehat{\phi}(\omega+1)|^{2} d \omega+\cdots \\
\|f\|_{L^{2}}^{2}= & \int_{0}^{1}\left|\left(\sum_{n} c_{n} e^{2 \pi i \omega n}\right)\right|^{2}\left(\cdots+|\widehat{\phi}(\omega-2)|^{2}+|\widehat{\phi}(\omega-1)|^{2}+|\widehat{\phi}(\omega)|^{2}\right. \\
& \left.+|\widehat{\phi}(\omega+1)|^{2}+\cdots\right) d \omega \\
\|f\|_{L^{2}}^{2}= & \int_{0}^{1}\left|\left(\sum_{n} c_{n} e^{2 \pi i \omega n}\right)\right|^{2}\left(\sum_{m}|\widehat{\phi}(\omega+m)|^{2}\right) d \omega,
\end{aligned}
$$

donde

$$
\Phi(\omega)=\sum_{m}|\widehat{\phi}(\omega+m)|^{2} .
$$

Pero

$$
\begin{aligned}
\int_{0}^{1}\left|\left(\sum_{n} c_{n} e^{2 \pi i \omega n}\right)\right|^{2} d \omega & =\left\langle\sum_{n} c_{n} e^{2 \pi i \omega n}, \sum_{k} c_{k} e^{2 \pi i \omega k}\right\rangle_{L^{2}([0,1])} \\
& =\sum_{n} \sum_{k} c_{n} \overline{c_{k}}\left\langle e^{2 \pi i \omega n}, e^{2 \pi i \omega k}\right\rangle_{L^{2}([0,1])} \\
& =\sum_{n}\left|c_{n}\right|^{2},
\end{aligned}
$$


entonces

$$
\begin{aligned}
A \sum_{n}\left|c_{n}\right|^{2} & \leq\left\|\sum_{n} c_{n} \phi(\cdot-n)\right\|^{2} \leq B \sum_{n}\left|c_{n}\right|^{2} \Leftrightarrow \\
A \sum_{n}\left|c_{n}\right|^{2} & \leq\|f\|_{L^{2}}^{2}=\left(\int_{0}^{1}\left|\sum_{n} c_{n} e^{2 \pi i \omega n}\right|^{2} d \omega\right)\left(\sum_{m}|\widehat{\phi}(\omega+m)|^{2}\right) \\
& \leq B \sum_{n}\left|c_{n}\right|^{2} \\
& \Leftrightarrow 0<A \leq \Phi(\omega)=\sum_{m}|\widehat{\phi}(\omega+m)|^{2} \leq B<\infty, \text { en c.t.p. }
\end{aligned}
$$

Definamos

$$
\widehat{\widetilde{\phi}}(\omega)=\frac{\widehat{\phi}(\omega)}{\sqrt{\Phi(\omega)}}
$$

Afirmación: $\sum_{k \in \mathbb{Z}}|\widehat{\widetilde{\phi}}(\omega+k)|^{2}=1$

En efecto, como $\widehat{\widetilde{\phi}}(\omega)=\frac{\widehat{\phi}(\omega)}{\sqrt{\Phi(\omega)}}$, entonces

$$
\begin{aligned}
|\widehat{\widetilde{\phi}}(\omega)|^{2} & =\frac{|\widehat{\phi}(\omega)|^{2}}{\Phi(\omega)} \\
\sum_{k}|\widehat{\widetilde{\phi}}(\omega+k)|^{2} & =\frac{\sum_{k}|\widehat{\phi}(\omega+k)|^{2}}{\Phi(\omega)}=1 .
\end{aligned}
$$

Luego, de acuerdo al Lema 2.2, $\sum_{k}|\widehat{\widetilde{\phi}}(\omega+k)|^{2}=1$, entonces $\{\tilde{\phi}(x-n)\}_{n \in \mathbb{Z}}$ es una base ortonormal. Sea

$$
\widetilde{V}_{0}=\left\langle\{\widetilde{\phi}(x-n)\}_{n \in \mathbb{Z}}\right\rangle .
$$

Por lo tanto, para todo $f \in \tilde{V}_{0}$,

$$
f(x)=\sum_{n} \tilde{f}_{n} \tilde{\phi}(x-n), \text { con } \tilde{f}_{n}=\left\langle f, \widetilde{\phi}_{0, n}\right\rangle \text { y } \sum_{n}\left|\tilde{f}_{n}\right|^{2}<\infty,
$$

esto es, $\widehat{f}(\omega)=\widetilde{v}(\omega) \widehat{\widetilde{\phi}}(\omega)$, tal que $\widetilde{v}(\omega)$ es una función periódica con período 1 y $\widetilde{v} \in L^{2}([0,1])$. Asimismo como

$$
\Phi(\omega)=\sum_{m}|\widehat{\phi}(\omega+m)|^{2}
$$

es una función periódica con periodo 1 , entonces

$$
\widehat{f}(\omega)=\widetilde{v}(\omega) \frac{\widehat{\phi}(\omega)}{\sqrt{\Phi(\omega)}}
$$


es decir,

$$
\widehat{f}(\omega)=\frac{\widetilde{v}(\omega)}{\sqrt{\Phi(\omega)}} \cdot \widehat{\phi}(\omega)=v(\omega) \widehat{\phi}(\omega)
$$

para alguna función periódica $v(\omega)$ con periodo 1 y $v \in L^{2}([0,1])$.

De este modo esto es equivalente a

$$
f(x)=\sum_{n \in \mathbb{Z}} f_{n} \phi(x-n),
$$

con $f_{n}=\left\langle f, \phi_{0, n}\right\rangle$ y $\sum_{n \in \mathbb{Z}}\left|f_{n}\right|^{2}<\infty$; de este modo $f \in V_{0}=\left\langle\{\phi(x-n)\}_{n \in \mathbb{Z}}\right\rangle$.

Desde que el argumento empleado nos permite regresar, se tiene que $\widetilde{V}_{0}=V_{0}$ y por lo tanto $\widetilde{V}_{j}=V_{j} \forall j=0, \pm 1, \pm 2 \pm \cdots$

La ondícula madre $\widetilde{\Psi}$ asociada con la función escala $\widetilde{\phi}$ viene dada, vía:

$$
\widehat{\widetilde{\Psi}}=e^{\pi i \omega} \overline{\widetilde{M}\left(\frac{\omega}{2}+\frac{1}{2}\right)} \widehat{\widetilde{\phi}}\left(\frac{\omega}{2}\right), \quad .
$$

donde $\widetilde{M}$ esta relacionada con $\widetilde{\phi}$ en la misma forma como $M$ esta relacionada con $\phi$. Además, desde que

$$
\widehat{\widetilde{\phi}}(\omega)=\widetilde{M}\left(\frac{\omega}{2}\right) \hat{\widetilde{\phi}}\left(\frac{\omega}{2}\right) \quad \text { y } \quad \widehat{\phi}(\omega)=M\left(\frac{\omega}{2}\right) \hat{\phi}\left(\frac{\omega}{2}\right)
$$

entonces se tiene

$$
\begin{aligned}
\widehat{\widetilde{\phi}}(\omega) & =\frac{\widehat{\phi}(\omega)}{\sqrt{\Phi(\omega)}} \Leftrightarrow \widetilde{M}\left(\frac{\omega}{2}\right) \widehat{\tilde{\phi}}\left(\frac{\omega}{2}\right)=\frac{M\left(\frac{\omega}{2}\right) \widehat{\phi}\left(\frac{\omega}{2}\right)}{\sqrt{\Phi(\omega)}} \\
& \Leftrightarrow \widetilde{M}\left(\frac{\omega}{2}\right) \frac{\widehat{\phi}\left(\frac{\omega}{2}\right)}{\sqrt{\Phi\left(\frac{\omega}{2}\right)}}=\frac{M\left(\frac{\omega}{2}\right) \widehat{\phi}\left(\frac{\omega}{2}\right)}{\sqrt{\Phi(\omega)}} \\
& \Leftrightarrow \widetilde{M}\left(\frac{\omega}{2}\right)=\sqrt{\frac{\Phi\left(\frac{\omega}{2}\right)}{\Phi(\omega)}} \cdot M\left(\frac{\omega}{2}\right) ;
\end{aligned}
$$

por lo tanto,

$$
\begin{aligned}
& \widehat{\widetilde{\Psi}}(\omega)=e^{\pi i \omega} \sqrt{\frac{\Phi\left(\frac{\omega}{2}+\frac{1}{2}\right)}{\Phi(\omega+1)}} \cdot M\left(\frac{\omega}{2}+\frac{1}{2}\right) \cdot \widehat{\widetilde{\phi}}\left(\frac{\omega}{2}\right) \\
& \widehat{\widetilde{\Psi}}(\omega)=e^{\pi i \omega} \sqrt{\frac{\Phi\left(\frac{\omega}{2}+\frac{1}{2}\right)}{\Phi(\omega+1)}} \cdot M\left(\frac{\omega}{2}+\frac{1}{2}\right) \cdot \frac{\widehat{\phi}\left(\frac{\omega}{2}\right)}{\sqrt{\Phi\left(\frac{\omega}{2}\right)}} \\
& \widehat{\widetilde{\Psi}}(\omega)=e^{\pi i \omega} \sqrt{\frac{\Phi\left(\frac{\omega}{2}+\frac{1}{2}\right)}{\Phi(\omega+1) \Phi\left(\frac{\omega}{2}\right)}} \cdot M\left(\frac{\omega}{2}+\frac{1}{2}\right) \cdot \widehat{\phi}\left(\frac{\omega}{2}\right)
\end{aligned}
$$




\section{REFERENCIAS BIBLIOGRÁFICAS}

[1] I. DAUBECHIES, Orthonormal bases of compactly Supported Wavelets, Comm. Pure Appl. Math. 41 (1988), 909-996.

[2] I. DAUBECHIES, Ten Lectures on Wavelets, CBMS-NSF Regional Conference Series in Applied Mathematics, SIAM publ. Philadelphia (1992)

[3] S.G. MALLAT, ., Multiresolution approximations and Wavelet ortonormal bases of $L^{2}(\mathbb{R})$, Trans. Amer. Math. Soc. 315 (1989), 69-87.

[4] C. CHUI, An-Introduction to Wavelets, Academic, Press, New York (1992). 